\title{
RESOLUCIÓN DEL PROBLEMA DIRECTO DE TOMOGRAFÍA SÍSMICA: TRAZADO DE RAYOS SÍSMICOS EN EL CLÚSTER DE PISAYAMBO
}

\author{
DIRECT PROBLEM RESOLUTION IN SEISMIC TOMOGRAPHY: SEISMIC RAY \\ TRACING IN PISAYAMBO CLÚSTER \\ Sebastián Araujo
}

Centro de Investigación en Modelamiento Ambiental, CIMA-UPS, Universidad Politécnica Salesiana, Av. 12 de Octubre N24-22 y Wilson, Telf. (593-2) 3962800, Quito, Ecuador

Autor para correspondencia: jaraujo@ups.edu.ec

Manuscrito recibido el 13 de marzo de 2012. Aceptado, tras revisión, el 12 de junio de 2012.

Nota: los datos utilizados en esta investigación fueron tomados del Instituto Geofísico de la Escuela Politécnica Nacional de Quito, Ecuador

\section{Resumen}

Este ensayo muestra los resultados prácticos de la aplicación del principio de Fermat para trazar rayos sísmicos a través de un medio heterogéneo. Para comenzar se muestran las ventajas de aplicar este principio físico en lugar de resolver las ecuaciones diferenciales de propagación de las ondas.

Para el experimento numérico se usa el programa FERMAT sobre sismos originados en el clúster de Pisayambo. FERMAT es un código de resolución del problema directo que hace uso del principio de tiempo mínimo de propagación de un rayo. Se muestran los resultados obtenidos para eventos a distintas profundidades del clúster. Estos rayos trazados pemiten obtener una visión más clara del proceso que puede ser el origen de los sismos.

Palabras claves: Pisayambo, sismología, problema directo, FERMAT.

\begin{abstract}
This work presents the practical results of the application of Fermat's principle on the seismic ray tracing in a heterogeneous medium. First, the advantages of applying this method over the resolution of differential equations of seismic wave propagation is shown.

Using FERMAT software, earthquakes originated in Pisayambo clúster are simulated. FERMAT software uses the Fermat's principle to solve the direct problem of seismic ray propagation minimizing the time parameter. The obtained results were calculated for different depths from the clúster. The seismic ray tracing technique offers the possibility to clarify the process originating earthquakes.
\end{abstract}

Keywords: Pisayambo, seismology, direct problem, FERMAT.

Forma sugerida de citar: Araujo, S. 2012. Resolución del problema directo de tomografía sísmica: trazado de rayos sísmicos en el clúster de Pisayambo. La Granja. Vol. 15(1): 48-54. ISSN: 1390-3799. 


\section{Introducción}

El clúster Pisayambo continúa hasta la presente fecha como el mayor generador de actividad sísmica dentro del territorio ecuatoriano. El último informe anual (Instituto Geofísico, 2010) del Instituto Geofísico de la Escuela Politécnica Nacional da cuenta de que el $20 \%$ del total de sismos de Ecuador se generaron dentro de esta zona. Se produjeron en ese año 255 temblores entre magnitudes 4,9 y 2,8. Dadas estas condiciones, el clúster representa una zona de gran riesgo sísmico para todas las provincias centrales del Ecuador. Es por esto que se hace necesario seguir afinando los estudios sobre la zona y poder definir correctamente el origen de semejante actividad.

Dentro de los métodos sismológicos que se pueden utilizar de forma novedosa para investigar Pisayambo se encuentra la tomografía. La base teórica de la tomografía impone la resolución de dos tipos de problemas: el directo y el inverso (Araujo, 2008). A continuación se expondrán los resultados de la resolución del primer problema utilizando sismos reales generados en Pisayambo. En términos técnicos, este proceso se los conoce como el trazado de rayos sísmicos a través de un medio de capas planas. Bien si los resultados pueden parecer limitados, este es un método muy útil para encontrar desviaciones del modelo de capas planas y poder asociarlas a fallas activas de la corteza (Bullen y Bolt, 1985; Shearer, 2009).

\section{Materiales y métodos}

\section{Softwares utilizados y pruebas con datos sintéticos}

La primera parte de la tomografía consistió en resolver el problema directo: dado un modelo de velocidades se calculó el tiempo de propagación de los rayos sísmicos a través del modelo de velocidades. Para el cálculo de los tiempos de propagación se supuso conocida la posición de origen del sismo, el hipocentro, y la posición de la estación donde fue detectado. Este tipo de problema directo se conoce como el trazado de rayos sísmicos entre dos puntos y para resolverlo se utiliza un algoritmo que soluciona la ecuación diferencial de propagación de la onda sísmica en dos dimensiones a través del método de Runge-Kutta (Margrave, 2003).

Se comienzó con la creación de un modelo de velocidades sintético en dos dimensiones y se probó el trazado de un haz de rayos que comienzaron en la superficie y se propagaron hacia el interior de la Tierra usando la función shootrayvxz (Margrave, 2003). El resultado puede verse en la Figura 1.

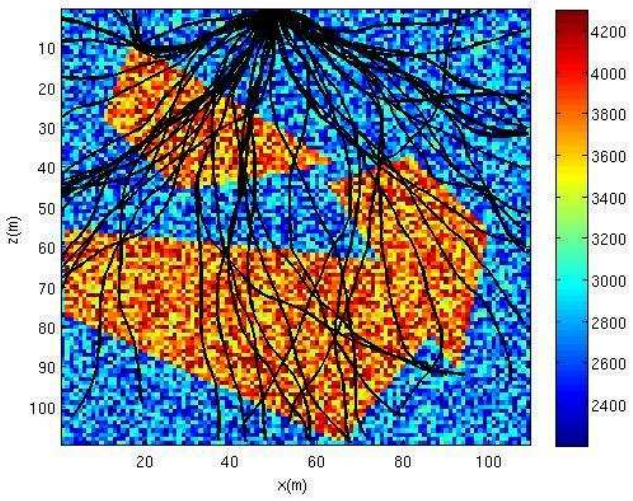

Figura 1. Un haz de rayos sísmicos se genera en la superficie y se propaga en profundidad a través de un modelo no lineal de velocidades. Solución utilizando CREWES.

El problema para la tomografía, sin embargo, consiste en trazar un rayo desde el hipocentro hasta la estación en la superficie. Para lograr este objetivo se usa también shootraytosurf (Margrave, 2003). En el ejemplo, se supone un evento que se localiza en el modelo de velocidades anterior en el punto $(40,80)$ y se lo propagó hasta una estación en la superficie de coordenadas $(90,0)$. Se debe tomar como parámetro para el trazado del rayo el ángulo de incidencia que para la geometría del problema se calcula como:

$$
\theta=90+\operatorname{tg}^{-1}\left(\frac{0-(-80)}{90-40}\right) .
$$

El resultado del trazado del rayo se muestra en la Figura 2.

En el caso real de los sismos de Pisayambo las ondas no se propagan en dos dimensiones sino en tres. Existen algoritmos de cálculo para la propagación de rayos sísmicos en tres dimensiones basados, como el caso anterior, en la resolución de la ecuación diferencial de propagación de la onda, la ecuación de la eikonal, utilizando diferencias finitas (Podvin y Lecomte, 1991); de hecho este tipo de algoritmos se 
utilizan en la resolución de problemas de tomografía (Monteiller et al., 2005). En este trabajo, sin embargo, se propone utilizar un algoritmo basado en el principio de Fermat o principio del tiempo mínimo (Araujo, 2010). Este algoritmo está implementado en un código de FORTRAN 90 y calcula la trayectoria del rayo buscando el tiempo más pequeño de propagación entre el hipocentro y la estación a través del método de optimización simplex (Nishi, 2001). Un ejemplo del funcionamiento del programa con datos sintéticos se muestra en la Figura 3.

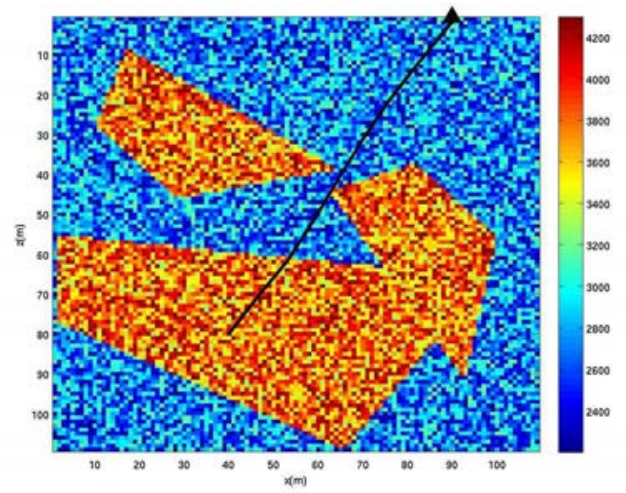

Figura 2. Propagación de un rayo sísmico desde el hipocentro hasta una estación en la superficie a través de un modelo de velocidades no lineal. Solución utilizando CREWES.

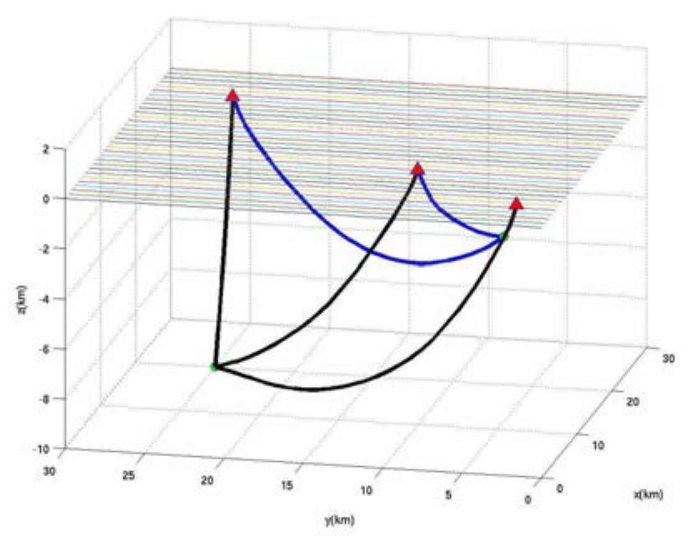

Figura 3. Aplicación de FERMAT a un ejemplo sintético. Dos hipocentros, puntos verdes, generan sendos sismos cuyos rayos se propagan hacia tres estaciones, triángulos rojos, situadas en la superficie. La esterilla de colores señala la superficie del terreno. El problema se resuelve en tres dimensiones.
En el presente proyecto se compiló el programa FERMAT usando gfortran-4.3 en una máquina ACER Aspire $4730 Z$ con procesador Intel Pentiun dual core que corre con una versión de LINUX OPENSUSE 11.1.

Es necesario escoger los parámetros adecuados para el funcionamiento de FERMAT. El cambio de estos parámetros mejora la calidad de la solución pero puede incrementar ostensiblemente el tiempo de cálculo. Haciendo un compromiso entre calidad y tiempo se tomaron los parámetros semiempíricos listados en la Tabla 1.

Tabla 1. Parámetros utilizados para el funcionamiento del programa FERMAT.

\begin{tabular}{cc}
\hline Parámetro & Valor \\
\hline n_1km & 1,0 \\
ndiv & 1 \\
maxloop & 100 \\
maxlocal_loop & 100 \\
simplex_range & 0,50 \\
simplex_min & 0,01 \\
simplex_init & 0,2 \\
nst & 10 \\
nevt & 1 \\
ngx,ngy,ngz & 774 \\
\hline
\end{tabular}

Con estos parámetros el tiempo de cálculo para cada evento está alrededor de los 30 minutos. El único parámetro que se modificó para cada cálculo es el que señala el número de estaciones nst.

El programa FERMAT necesita también de un modelo de velocidades sobre el cual trabajar. Como lo que aquí se trata es de resolver el problema directo, este modelo se escoge fijo de capas planas idéntico al utilizado en la relocalización con HYPODD (Araujo et al., 2009). Con estos datos de velocidad se formó una malla para los nodos en tres dimensiones que se muestran en la Tabla 2.

Tabla 2. Parámetros que indican las dimensiones de la malla $180 \times 240 \mathrm{~km}^{2}$. La escala vertical va desde los 5000 msnm hasta los $20 \mathrm{~km}$ de profundidad.

\begin{tabular}{ccccccc}
\hline 0.0 & 30.0 & 60.0 & 90.0 & 120.0 & 150.0 & 180.0 \\
\hline 0.0 & 40.0 & 80.0 & 120.0 & 160.0 & 200.0 & 240.0 \\
\hline-5.0 & 0.0 & 5.0 & 20.0 & & & \\
\hline
\end{tabular}


El modelo por tanto exploró posiciones desde los $5000 \mathrm{msnm}$ hasta $20 \mathrm{~km}$ de profundidad. Los $5 \mathrm{~km}$ sobre la superficie cubrió las posiciones de todas las estaciones; los $20 \mathrm{~km}$ se escogieron como cota máxima debido a los resultados obtenidos en la relocalización por dobles diferencias de los datos usados (Araujo et al., 2009).

\section{Aplicación de FERMAT a los datos de Pisayambo}

Los sismos sobre los cuales se van a trazar los rayos pertenecen a la red de estaciones del IG de la EPN, son aquellos que han sido relocalizados con HYPODD (Araujo et al., 2009). Son un total de 137 eventos de los cuales se han escogido en orden cronológico 11 eventos, cada uno a $2 \mathrm{~km}$ de profundidad del anterior. Así el primer evento es casi superficial a $0 \mathrm{~km}$ y el el último está a $20 \mathrm{~km}$. El objetivo es observar como cambia la propagación de los rayos a distintas profundidas y así visualizar posibles anomalías.

Comenzando desde los eventos más superficiales a $0 \mathrm{~km}$ (Figura 4 ) y a $2 \mathrm{~km}$ (Figura 5) se puede constatar que el único rayo que tiene propagación directa desde el hipocentro hasta la estación es el que se traza hacia la estación PISA. Todos los demás rayos hacen un camino en profundidad y señalan una anomalía a los $5 \mathrm{~km}$ de profundidad. El hecho de que no haya una propagación directa para los rayos del resto de las estaciones indica la fuerte irregularidad estructural de la parte más superficial de la corteza que no se corresponde con el modelo de capas planas que se usa como punto de partida.

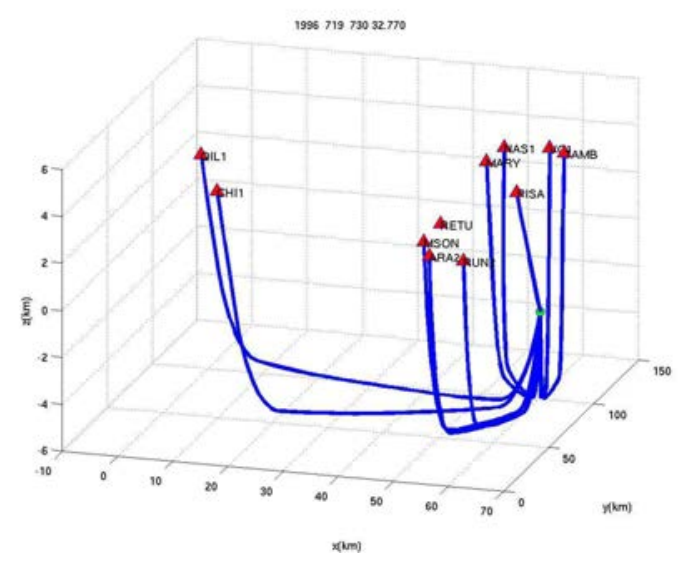

Figura 4. Evento a 0,994 km de profundidad.

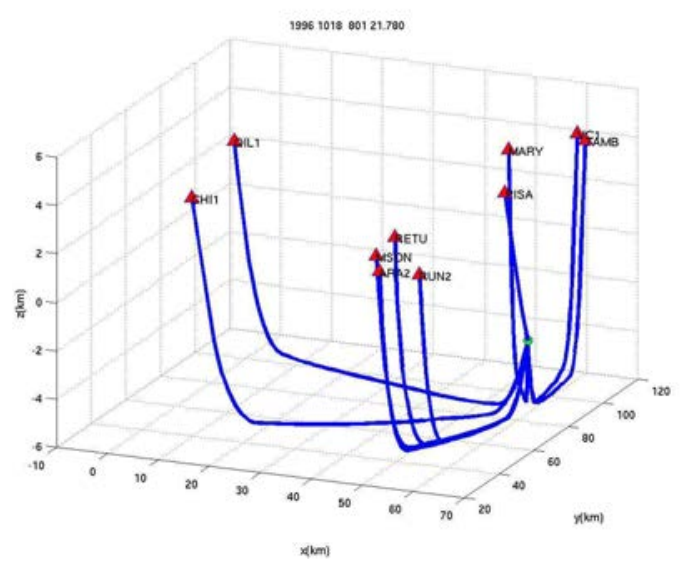

Figura 5. Evento a 2,047 km de profundidad.

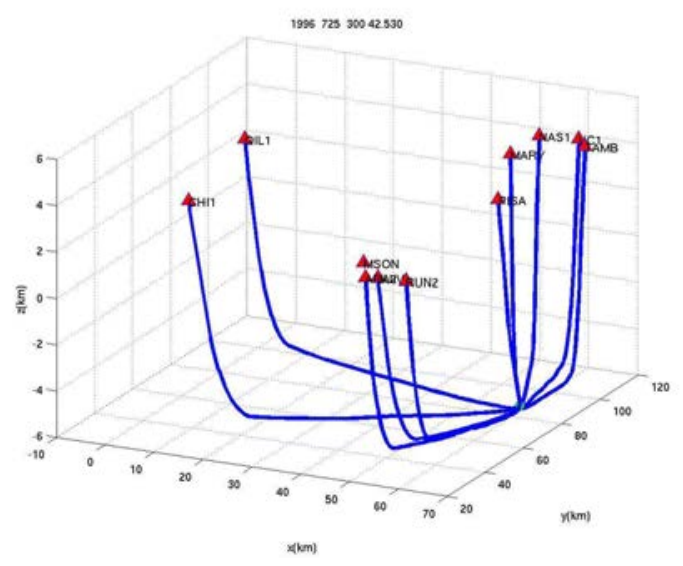

Figura 6. Evento a 4,796 km de profundidad.

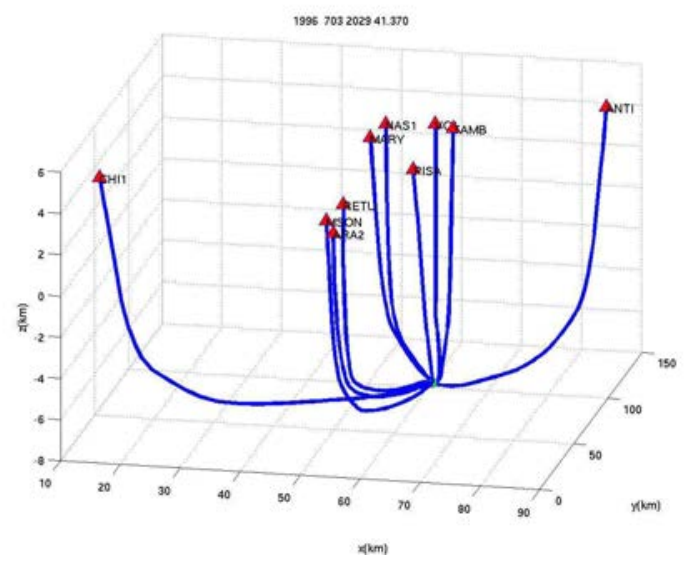

Figura 7. Evento a 6,517 km de profundidad.

LA GRANJA, Revista de ciencias de la vida, 15(1) 2012: 48-54.

(C) 2012, Universidad Politécnica Salesiana, Ecuador. 
En las Figuras 6 y 7 donde se muestran rayos de eventos situados a $4 \mathrm{~km}$ y $6 \mathrm{~km}$, respectivamente, se aprecia un comportamiento similar, los rayos no se propagan rectilíneamente debido a la discontinuidad existente alrededor de los $5 \mathrm{~km}$.

En el evento a $8 \mathrm{~km}$ de profundidad (Figura 8) hay un resultado de interés debido a que se muestran los rayos propagados hacia la red temporal instalada sobre el volcán Cotopaxi. Estas estaciones se señalan con letras minúsculas para diferenciarlas de las estaciones permanentes del Instituto Geofísico que se escriben con mayúsculas. Son importantes, sobre todo, los rayos trazados hacia las estaciones refu, hiel, VC1 y moro que están precisamente alrededor del cono volcánico del Cotopaxi. Los rayos en apariencia verticales entre los $5 \mathrm{~km}$ hasta casi los 6 $\mathrm{km}$ sobre la superficie contornean el cono y la estructura subyacente.

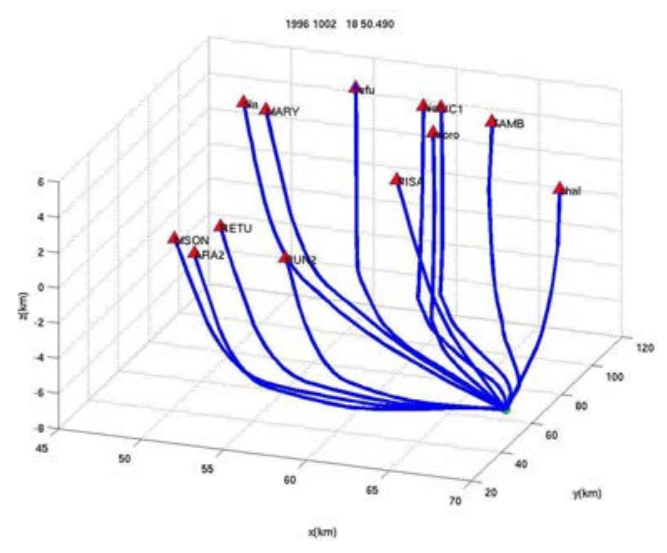

Figura 8. Evento a 7,470 km de profundidad.

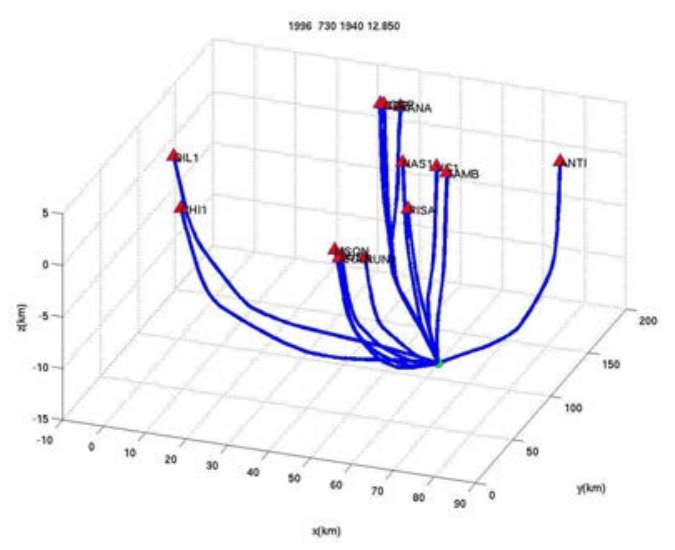

Figura 9. Evento a 10,323 km de profundidad.
A medida que el estudio progresa en profundidad los rayos tienden a sobrepasar la discontinuidad de la malla y no muestran cambios inesperados en sus trayectorias de propagación (Figuras 9, 10 y 11).

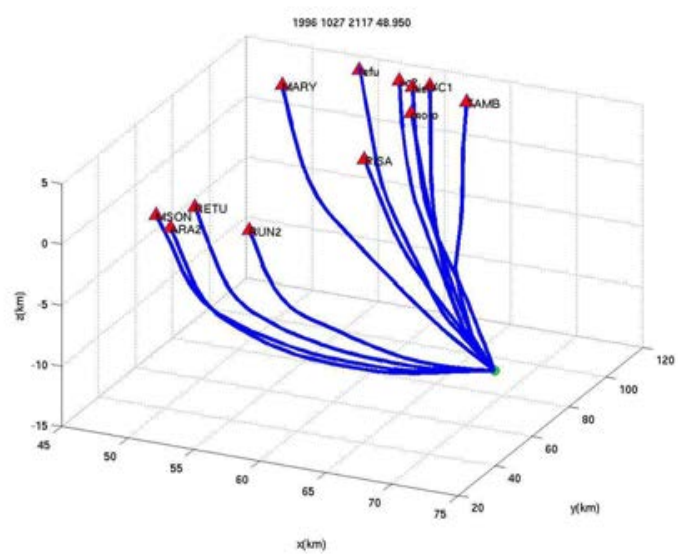

Figura 10. Evento a 11,419 km de profundidad.

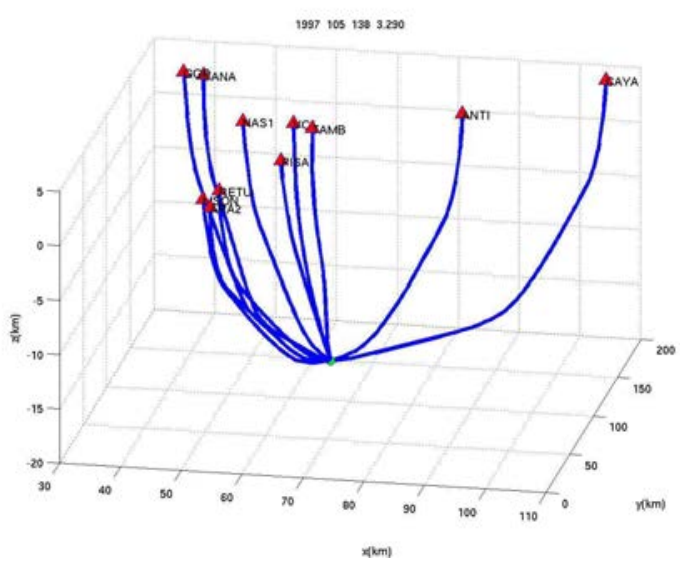

Figura 11. Evento a $14,723 \mathrm{~km}$ de profundidad.

Asimismo, para los eventos más profundos del clúster a $16 \mathrm{~km}, 18 \mathrm{~km}$ y $20 \mathrm{~km}$ que se grafican en la Figuras 12, 13 y 14, respectivamente, tampoco aparecen discontinuidades importantes en los rayos. 


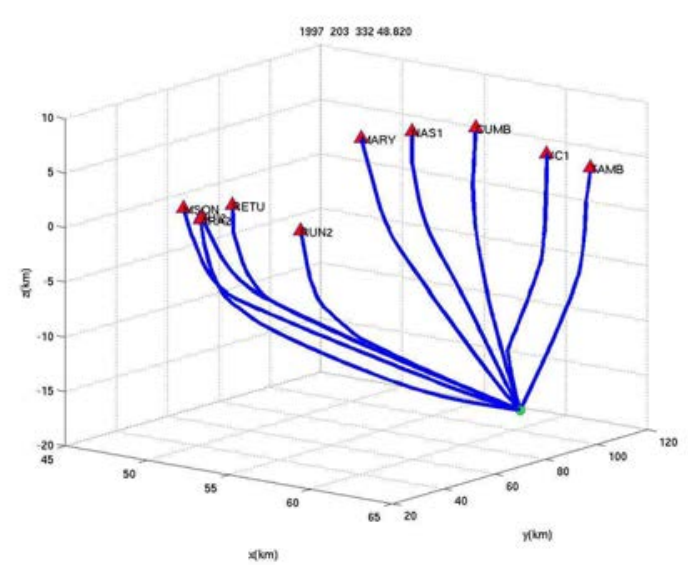

Figura 12. Evento a $15,220 \mathrm{~km}$ de profundidad.

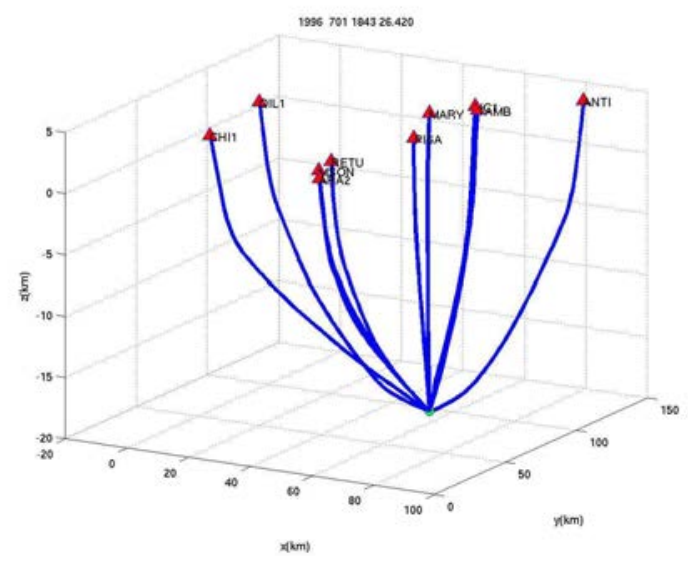

Figura 13. Evento a $18,307 \mathrm{~km}$ de profundidad.

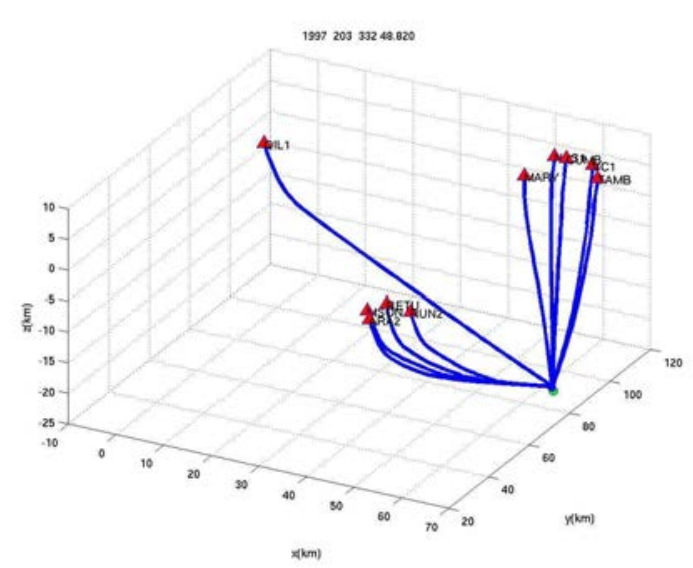

Figura 14. Evento a 20,962 km de profundidad.

\section{Discusión y conclusiones}

De las pruebas realizadas con FERMAT sobre los sismos de Pisayambo propagándose a través del modelo de capas planas, no se aprecia en principio ninguna discontinuidad importante salvo para los eventos más superficiales que muestran un cambio abrupto en la propagación de los rayos alrededor de los $5 \mathrm{~km}$ de profundidad. Este cambio en la trayectoria de propagación de los rayos sísmicos es claro debido al alto contraste en el modelo de velocidades incial a esa profundidad.

En efecto, el modelo de capas planas cambia la velocidad de propagación de la onda $\mathrm{P}$ de 3,32 $\mathrm{km} / \mathrm{h}$ a $5,90 \mathrm{~km} / \mathrm{h}$. Esta anomalía es claramente detectada por FERMAT.

Se ha realizado, por tanto, la prueba sobre eventos reales, de que el trazado de rayos sísmicos permite detectar los cambios entre las capas superficiales. El siguiente paso en la investigación será la perturbación del modelo de capas planas lo cual corresponderá a la resolución del problema inverso de la tomografía.

\section{Agradecimiento}

Se agradece al Instituto Geofísico de la Escuela Politécnica Nacional por los datos proporcionados para el presente estudio.

\section{Referencias}

Araujo, S. 2008. Solución probabilística del problema inverso. En: Memorias del XI Encuentro de Matemáticas y sus aplicaciones.

Araujo, S. 2010. Aplicación del principio de fermat a la tomografía sísmica. parte 1: Fundamentos físicos. La Granja.

Araujo, S., L. Troncoso y M. Ruiz. 2009. Relocalización por dobles diferencias del clúster sísmico de pisayambo. La Granja, 10(2): 27-34.

Bullen, K. y B. Bolt. 1985. An introduction to the theory of seismology. Cambridge University Press. 
Instituto Geofísico. 2010. Informe sísmico para el año 2010. Informe técnico, Escuela Politécnica Nacional.

Margrave, G. 2003. Numerical Methods of Exploration Seismology with algorithms in MATLAB. CREWES.

Monteiller, V., J. Got y P. J.Virieux. 2005. An eficient algorithm for Doubble diference tomography and location in heterogeneous media, with application to the Kilauea volcano. J. Geophys. Res.,110, B12306.
Nishi, K. 2001. A three-dimensional robust seismic ray tracer for volcanic regions. Earth Planets Space, 53: 101-109.

Podvin, P. y I. Lecomte. 1991. Finite difference computation of traveltimes in very contrasted velocity models: a massively parallel approach and its associated tools. Geophys. J. Int., 105, págs. 271-284.

Shearer, P. 2009. Introduction to seismology. Cambridge University Press. Cambridge University Press. 